\title{
Prospective association of a genetic risk score and lifestyle intervention with cardiovascular morbidity and mortality among individuals with type 2 diabetes: the Look AHEAD randomised controlled trial
}

\author{
The Look AHEAD Research Group
}

Received: 18 November 2014 / Accepted: 7 April 2015 /Published online: 14 May 2015

(C) Springer-Verlag Berlin Heidelberg 2015

\begin{abstract}
Aims/hypothesis Both obesity and genetics contribute to cardiovascular disease (CVD). We examined whether a genetic risk score (GRS) prospectively predicted cardiovascular morbidity and mortality among overweight/obese individuals with type 2 diabetes and whether behavioural weight loss could diminish this association.

Methods Look AHEAD (Action for Health in Diabetes) is a randomised controlled trial to determine the effects of intensive lifestyle intervention (ILI), including weight loss and physical activity, relative to diabetes support and education, on cardiovascular outcomes among overweight/obese individuals with type 2 diabetes. Of the participants, 4,016 provided consent for genetic analyses and had DNA samples passing quality control procedures. These secondary data analyses focused on whether a GRS derived from 153 single nucleotide polymorphisms (SNPs) associated with coronary artery disease in the most recent genome-wide association study predicted cardiovascular morbidity and mortality over a median of 9.6 years of follow-up, and whether ILI would diminish this association.
\end{abstract}

The complete author list for the Look AHEAD Research Group is provided in the Acknowledgements. The complete list of Look AHEAD investigators is provided in the electronic supplementary material (ESM).

Electronic supplementary material The online version of this article (doi:10.1007/s00125-015-3610-z) contains peer-reviewed but unedited supplementary material, which is available to authorised users.

The Look AHEAD Research Group jeanne_mccaffery@brown.edu

1 c/o J. McCaffery, Weight Control and Diabetes Research Center, The Miriam Hospital and Brown Medical School, 196 Richmond Street, Providence, RI 02903, USA
Results The GRS significantly predicted the primary composite endpoint of death from cardiovascular causes, nonfatal myocardial infarction, nonfatal stroke, or hospitalisation for angina in the full sample (HR, 95\% CI per 1 SD increase in GRS: $1.19[1.10,1.28])$ and among individuals with no known history of CVD at baseline (HR 1.18 [95\% CI 1.07, 1.30]). In no case did ILI significantly alter this association.

Conclusions/interpretation A GRS comprised of SNPs significantly predicts cardiovascular morbidity and mortality over 9.6 years of follow-up in Look AHEAD. Lifestyle intervention did not alter the genetic association.

Clinical Trial Registration: NCT00017953; NCT01270763

Keywords Cardiovascular disease · Genetics · Lifestyle intervention · Myocardial infarction · Obesity $\cdot$ Stroke . Type 2 diabetes $\cdot$ Weight loss

$\begin{array}{ll}\begin{array}{l}\text { Abbreviations } \\ \text { CABG }\end{array} & \begin{array}{l}\text { Coronary artery bypass grafting } \\ \text { CAD }\end{array} \\ \text { CARDIoGRAMplusC4D } & \begin{array}{l}\text { Coronary Artery disease } \\ \text { Genome-wide Replication and } \\ \text { Meta-analysis (CARDIoGRAM) } \\ \text { consortium plus the Coronary }\end{array} \\ & \text { Artery Disease (C4D) } \\ & \text { consortium } \\ & \text { Cardiovascular disease } \\ \text { CVD } & \text { Diastolic blood pressure } \\ \text { DBP } & \text { Diabetes support and education } \\ \text { DSE } & \text { (arm of the Look AHEAD trial) } \\ & \text { False discovery rate } \\ \text { FDR } & \text { Genetic risk score } \\ \text { GRS } & \end{array}$




$\begin{array}{ll}\text { ILI } & \begin{array}{l}\text { Intensive lifestyle intervention } \\ \text { (arm of the Look AHEAD trial) } \\ \text { Institute of Translational Medi- } \\ \text { cine and Therapeutics (ITMAT)- } \\ \text { Broad-CARe genotyping array }\end{array} \\ \text { IBC } & \begin{array}{l}\text { Linkage disequilibrium } \\ \text { Action for Health in Diabetes } \\ \text { study }\end{array} \\ \text { LD } & \text { Multi-dimensional scaling } \\ \text { Look AHEAD study } & \text { Percutaneous coronary } \\ \text { MDS } & \text { intervention } \\ \text { PCI } & \text { Systolic blood pressure } \\ \text { SBP } & \text { Single nucleotide polymorphism }\end{array}$

\section{Introduction}

Genetic and environmental factors collectively contribute to the pathogenesis of cardiovascular disease (CVD), which remains the leading cause of death both in the USA [1] and globally [2]. Death rates attributable to CVD in the USA have declined secondary to improvements in factors heavily influenced by the environment, including cigarette smoking. However, this favourable trend in mortality rates has been at least partially offset by increases in obesity and type 2 diabetes [3], potent risk factors for CVD [1]. At the same time, genome-wide association studies have identified single nucleotide polymorphisms (SNPs) present in the general population associated with prevalent coronary artery disease (CAD) [4]. Several studies have examined whether subsets of these SNPs, alone or in combination, are prospectively associated with CVD morbidity and mortality [5-13], with seemingly greater success with more comprehensive SNP panels [8, 13]. Yet, no studies have examined the most comprehensive list of SNPs to determine the association with CVD outcomes and whether such genetic risk may be mitigated by behavioural weight loss.

The Look AHEAD (Action for Health in Diabetes) study is a multi-centre trial that tested whether a randomly assigned intensive lifestyle intervention (ILI) designed to bring about weight loss and increased fitness would reduce the incidence of CVD compared with diabetes support and education (DSE) that did not have weight loss or physical activity goals. Look AHEAD participants in the ILI arm lost significantly more weight and showed greater improvement in fitness, waist circumference, levels of HDL-cholesterol, triacylglycerol, systolic blood pressure (SBP) and diastolic blood pressure (DBP), and indices of diabetes control (including diabetes medication use, $\mathrm{HbA}_{1 \mathrm{c}}$ and levels of fasting glucose) compared with participants in the DSE arm after 1 year [14]. Despite this, after a median of 9.6 years follow-up Look AHEAD participants in the two study arms showed similar rates of cardiovascular morbidity and mortality [15].
It is currently unclear why the behavioural intervention, which amounted to a favourable change in environment, failed to provide a CVD benefit to participants of Look AHEAD. In this paper, we asked whether genetic factors may have contributed to the primary and secondary study endpoints of CVD in Look AHEAD and whether the efficacy of the intervention may have varied by genetic risk. To address this question we calculated a genetic risk score (GRS) based upon the most recent genome-wide association search for common variants contributing to CAD conducted by the Coronary Artery Disease Genome-wide Replication and Meta-analysis (CARDIoGRAM) consortium plus the Coronary Artery Disease (C4D) consortium, or the CARDIoGRAMplusC4D consortium, which reported in 2013 [4]. The 153 SNPs identified from populations of European or South Asian descent as associated with CAD at a false discovery rate of less than $5 \%$ were included in the GRS. This GRS was examined for a prospective association with CVD morbidity and mortality defined as the primary and secondary outcomes of Look AHEAD. It was further determined whether any observed associations of the GRS with CVD outcomes were diminished by randomised weight-loss intervention.

\section{Methods}

\section{Study cohort}

The Look AHEAD study is a 16 centre, randomised controlled trial with the primary goal of determining whether randomisation to ILI, including diet and physical activity, reduces cardiovascular morbidity and mortality relative to DSE alone among overweight or obese individuals with type 2 diabetes. The design and methods of Look AHEAD have been reported elsewhere [16], as have the baseline characteristics of the randomised cohort [17]. Briefly, 5,145 ethnically diverse overweight and obese individuals with type 2 diabetes aged 45-76 years were randomly allocated to either the ILI or DSE arm. Participants in both arms were provided with one session of education on diabetes and cardiovascular risk factors. In addition, ILI patients received an intensive lifestyle programme, combining diet modification and increased physical activity, designed to produce an average of $7 \%$ weight loss and maintain these weight losses. The DSE group received the option of attending three sessions per year on nutrition, physical activity and social support, with no explicit weight loss goals. Medications were managed by participants' personal physicians who were not involved in the trial.

Of the 5,125 Look AHEAD participants, 4,322 provided consent for genetic analyses. Participants who did not provide genetic consent were more likely to be grouped as AfricanAmerican, Hispanic, female, more highly educated and not 
dyslipidaemic [18]. After quality control procedures and exclusion of related individuals (see Genotyping and Quality Control section), 4,016 participants were included in the present analyses.

Randomisation occurred from August 2001 to April 2004. Data contributing to the current analyses were collected until 14 September 2012 when the ILI was stopped because of a lack of effect on the incidence of major CVD events [15]. The median follow-up was 9.6 years (interquartile range $8.9-10.3)$. Less than $4 \%$ of all patients randomly assigned to a study group had been lost to follow-up.

Look AHEAD was approved by local Institutional Review Boards, including genetic analyses. All participants provided informed consent.

\section{Measures}

Baseline CVD and cardiovascular risk factors CVD history at baseline was defined by self-report of a prior history of heart attack, stroke, bypass surgery, stent placement, angioplasty, carotid endarterectomy, angioplasty of lower extremity artery, aortic aneurysm repair, or heart failure or congestive heart failure. Weight (in $\mathrm{kg}$ ) and height (in $\mathrm{cm}$ ) were measured in duplicate using a digital scale and stadiometer, respectively. Blood pressure was measured in duplicate using an automated device. Averages of the duplicate measures of height, weight and blood pressure were used in analyses. Blood work was completed after an overnight fast and was analysed by Northwest Lipid Research Laboratories (University of Washington, Seattle, WA, USA) using standardised laboratory procedures. LDL-cholesterol levels were estimated using the Friedewald equation. Participants brought all prescription medications to their assessments.

Cardiovascular endpoints During annual visits and telephone calls every 6 months, staff members who were unaware of study group assignments queried participants about all medical events and hospitalisations. Hospital and other records were reviewed for potential cardiovascular events, with adjudication according to standard criteria by a central review committee who were unaware of study group assignments.

The primary composite CVD outcome for the Look AHEAD study was cardiovascular death or the first occurrence of nonfatal myocardial infarction, nonfatal stroke, or hospitalisation for angina [15]. Secondary outcomes included: (1) cardiovascular death, nonfatal myocardial infarction or nonfatal stroke; (2) all-cause mortality, myocardial infarction, stroke, or hospitalisation for angina; and (3) all-cause mortality, myocardial infarction, stroke, hospitalisation for angina, coronary artery bypass grafting $(\mathrm{CABG})$, percutaneous coronary intervention (PCI), hospitalisation for heart failure, or peripheral vascular disease.

\section{Genotyping and quality control}

We attempted to genotype DNA samples from 4,322 individuals with the MetaboChip (Illumina, San Diego, CA, USA). We also genotyped 35 unrelated samples from the International HapMap Project ( $n=15$ for CEPH [Utah residents with ancestry from northern and western Europe; CEU]; $n=4$ for Han Chinese in Beijing, China [CHB]; $n=4$ for Japanese in Tokyo, Japan [JPT]; $n=3$ for Mexican ancestry in Los Angeles, California [MEX] and $n=9$ for Yoruba in Ibadan, Nigeria [YRI]) to serve as anchors in analyses of genetic ancestry. We removed samples that failed genotyping ( $>5 \%$ missing data), blind duplicate samples, samples displaying sex inconsistencies, and samples displaying a high degree of discordance with genotyping available from the Institute of Translational Medicine and Therapeutics (ITMAT)-BroadCARe (IBC) CVD array [19]. This reduced the available sample size to 4,258 .

To address issues related to population structure and latent familial clustering, we first created a subset of MetaboChip (Illumina) SNPs (177,142 out of 196,725) after removing non-autosomal SNPs, SNPs that could not be reliably mapped to the reference genome (see annotation information available at www.sph.umich.edu/csg/kang/MetaboChip/, accessed 3 March 2015), SNPs in known regions of long-range linkage disequilibrium (LD) in European populations (such as the MHC) [20], SNPs with high discordance compared with genotyping available on the IBC CVD array, and SNPs with a high proportion of missing data $(>5 \%)$. We then pruned this set of SNPs to create a final set of SNPs $(67,592)$ in relative linkage equilibrium using the pair-wise pruning procedure implemented in PLINK (http://pngu.mgh.harvard.edu/ purcell/plink/) [21], assuming an $r^{2}$ threshold of 0.2 , a window size of 100 SNPs and shifting the window 25 SNPs at a time.

By using the LD-pruned subset of SNPs, we then conducted a multi-dimensional scaling (MDS) analysis using KING (http://people.virginia.edu/ wc9c/KING/kingpopulation.html [22]). We removed samples of Asian ancestry (due to a small number of participants in Look AHEAD) and outlying samples based on the MDS analysis, reducing the sample size to 4,121 (ESM Fig. 1). We then estimated European, West African, and Native American admixture proportions $(K=3)$ using ADMIXTURE, version 1.23 (https://www.genetics.ucla.edu/software/admixture/ [23]), using these estimates within REAP, version 1.2 (http://faculty.washington.edu/tathornt/software/REAP/ [24]) to estimate pair-wise kinship coefficients accounting for the multi-ethnic structure of the Look AHEAD population. Based on the kinship coefficient estimates from REAP, we created an unrelated subset of participants using the Pedigree Reconstruction and Identification of a Maximum Unrelated Set (PRIMUS) software programme (https://primus.gs. washington.edu/ [25]), based on a kinship coefficient 
threshold of 0.025 , resulting in the final sample size of 4,016 individuals.

In addition to the 45 susceptibility loci identified by the CARDIoGRAMplusC4D consortia [4], the consortia also undertook a false discovery rate (FDR) analysis, reporting an additional 104 SNPs associated with CAD at a FDR threshold of 5\%. This expanded set of 153 SNPs was estimated to account for $10.6 \%$ of the additive genetic variance for CAD based on a multifactorial liability threshold model [26]. Descriptive statistics for the 153 SNPs in Look AHEAD are presented in ESM Table 1. All SNPs were directly genotyped on the MetaboChip (Illumina) and did not exhibit significant deviation from Hardy-Weinberg equilibrium in the European subgroup of the Look AHEAD cohort ( $p>0.002$ for all SNPs).

To address the minimal degree of sporadic missing genotypes in constructing the GRS, we imputed missing genotypes using BEAGLE, version 3.0 (http://faculty. washington.edu/browning/beagle/beagle.html [27]), performing the imputation separately for the subsets of European, African-American and Hispanic/Native American participants. Across SNPs, 122/153 (79.7\%) had no missing data, with a maximum no-call rate of 1.5\%. Across individuals, 3,778/4,016 (94.1\%) had no missing data, with a maximum missing rate of $2.6 \%$. All results are presented in terms of a weighted GRS, which sums the risk alleles for each of the 153 SNPs assuming an additive coding multiplied by the log-odds ratio (natural logarithm) reported from the heritability analyses of the CARDIoGRAMplusC4D consortia (ESM Table 1). For imputed SNPs, we summed the log-odds ratio estimates multiplied by the corresponding genotype probability estimated by BEAGLE.

\section{Statistical modelling}

All statistical analyses consisted of secondary data analyses in the Look AHEAD cohort. We evaluated the relationship between the GRS and CVD risk factors (BMI, LDL, HDL, $\log _{\mathrm{e}}$ triacylglycerol, SBP, DBP and $\log \mathrm{HbA}_{1 \mathrm{c}}$ ) at baseline using linear models. To address treatment effects of antihypertensive medications on SBP and DBP, lipid-lowering medications on LDL, HDL and triacylglycerol, and insulin use on $\mathrm{HbA}_{1 \mathrm{c}}$, we used censored normal linear models [28], treating the observed values as right-censored (SBP, DBP, LDL, $\log$ triacylglycerol and $\log \mathrm{HbA}_{1 \mathrm{c}}$ ) or left-censored (HDL) for those on treatment. All models included additional adjustments for age, sex, clinical site, and the top three MDS vectors to address population stratification.

The relationship between the GRS and CVD outcomes was estimated using conditional Cox proportional hazards regression models including a gamma frailty term to account for dependence between participants at the same clinical site
[29]. Note that with conditional models, as opposed to marginal population-averaged models, effects are interpreted as a comparison between two participants at the same clinical site. The presented results consider two sets of models. First, we fitted models evaluating the main effect of the GRS on CVD outcomes in the combined Look AHEAD cohort, adjusting for the main effect of the intervention arm. Second, we considered models including an interaction term between the GRS and the intervention arm. All models began with additional covariate adjustments for age, sex and the top three MDS vectors. We also considered expanded models including adjustments for cardiovascular risk factors, use of antihypertensive and lipid-lowering medications, and use of aspirin. Unless otherwise indicated, all analyses were performed using the R Statistical Computing Environment (http://www.r-project.org/ [30]).

We conducted simulations assuming an exponential baseline hazard [31] to evaluate our power to detect an association between the GRS and CVD morbidity and mortality, as well as to detect an interaction between the intervention arm and the GRS. Assuming a follow-up period of 9.6 years and an alpha level of 0.05 , we had greater than $80 \%$ power to detect a hazard ratio of 1.12 per $1 \mathrm{SD}$ increase in the GRS in the full sample $(n=4,016)$. To estimate power for the interaction between the GRS and treatment arm with respect to CVD morbidity and mortality, hypothetical scenarios were created in which the ILI intervention suppressed the effect of the GRS on CVD morbidity and mortality relative to the DSE intervention with various effect sizes in each arm. For statistical power to be $>80 \%$ in the full Look AHEAD sample, there would need to be a strong association of the GRS in the DSE arm (HR per $1 \mathrm{SD}$ increase in GRS of $\geq 1.25$ ), with almost no effect in the ILI arm (HR $<1.05$ per $1 \mathrm{SD}$ increase in the GRS). Overall, we had excellent power to detect the estimated main effect of the GRS and, moreover, had power to detect a relatively large interaction of the GRS with the intervention arm.

\section{Results}

\section{Baseline characteristics}

Demographic characteristics of the genetic subsample at baseline are included in Table 1. The proportion of participants with a history of CVD or taking diabetes, hypertension or lipid-lowering medications were similar across a randomly assigned treatment arm, as were diabetes duration, $\mathrm{HbA}_{1 \mathrm{c}}$ level and cardiovascular risk factors. The CAD GRS was also equally distributed by treatment arm.

The CAD GRS was significantly associated with self-report of CVD history at baseline across all participants (Table 2), an effect that persisted in the subgroups of non-Hispanic white as well as Hispanic and Native 
Table 1 Baseline characteristics of Look AHEAD participants included in the genetic sub-study

\begin{tabular}{|c|c|c|c|}
\hline Characteristic & $\begin{array}{l}\text { ILI } \\
n=2,047\end{array}$ & $\begin{array}{l}\text { DSE } \\
n=1,969\end{array}$ & $p$ value \\
\hline Age (years) & $58.9 \pm 6.8$ & $59.1 \pm 6.8$ & 0.217 \\
\hline Female sex & $1,187(58.0)$ & $1,126(57.2)$ & 0.630 \\
\hline Self-reported race/ethnicity & & & 0.545 \\
\hline Non-Hispanic white & $1,432(70.0)$ & $1,407(71.5)$ & \\
\hline African-American & $310(15.1)$ & $295(15.0)$ & \\
\hline Hispanic & $227(11.1)$ & $187(9.5)$ & \\
\hline Native American & $42(2.1)$ & $41(2.1)$ & \\
\hline Other & $36(1.8)$ & $39(2.0)$ & \\
\hline Ancestral group ${ }^{\mathrm{a}}$ & & & 0.216 \\
\hline Non-Hispanic white & $1,410(68.9)$ & $1,397(70.9)$ & \\
\hline African-American & $330(16.1)$ & $313(15.9)$ & \\
\hline Hispanic/Native American & $307(15.0)$ & $259(13.2)$ & \\
\hline History of CVD & $305(14.9)$ & $272(13.8)$ & 0.349 \\
\hline Use of diabetes medications & $1,796(87.7)$ & $1,716(87.2)$ & 0.586 \\
\hline Use of insulin & $382(18.7)$ & $378(19.2)$ & 0.694 \\
\hline Use of hypertension medications ${ }^{\mathrm{b}}$ & $1,560(76.2)$ & $1,450(73.8)$ & 0.074 \\
\hline Use of lipid-lowering medications ${ }^{\mathrm{c}}$ & $1,072(52.4)$ & $1,050(53.4)$ & 0.586 \\
\hline Aspirin use & & & 0.378 \\
\hline Never & $896(43.8)$ & $860(43.7)$ & \\
\hline Sometimes & $192(9.4)$ & $208(10.6)$ & \\
\hline Every day & $954(46.6)$ & $892(45.3)$ & \\
\hline Unknown & $5(0.2)$ & $9(0.5)$ & \\
\hline Current smoking & $96(4.7)$ & $76(3.9)$ & 0.228 \\
\hline Duration of diabetes (years) & $5(2,10)$ & $5(2,10)$ & 0.500 \\
\hline Weight (kg) & $101.7 \pm 19.5$ & $102.2 \pm 18.7$ & 0.411 \\
\hline BMI $\left(\mathrm{kg} / \mathrm{m}^{2}\right)$ & $36.1 \pm 6.0$ & $36.2 \pm 5.8$ & 0.528 \\
\hline Waist circumference $(\mathrm{cm})$ & $114.5 \pm 14.2$ & $114.8 \pm 13.6$ & 0.441 \\
\hline $\mathrm{HbA}_{1 \mathrm{c}}(\%)$ & $7.2 \pm 1.1$ & $7.3 \pm 1.2$ & 0.630 \\
\hline $\mathrm{HbA}_{1 \mathrm{c}}(\mathrm{mmol} / \mathrm{mol})$ & $55.7 \pm 12.4$ & $55.9 \pm 12.7$ & 0.630 \\
\hline \multicolumn{4}{|l|}{ Blood pressure (mmHg) } \\
\hline Systolic & $128.9 \pm 17.3$ & $129.9 \pm 17.1$ & 0.056 \\
\hline Diastolic & $70 \pm 9.4$ & $70.4 \pm 9.6$ & 0.249 \\
\hline \multicolumn{4}{|l|}{ Cholesterol (mmol/l) } \\
\hline LDL & $2.9 \pm 0.8$ & $2.9 \pm 0.8$ & 0.697 \\
\hline HDL & $1.1 \pm 0.3$ & $1.1 \pm 0.3$ & 0.558 \\
\hline Triacylglycerol (mmol/l) & $1.8(1.2,2.5)$ & $1.7(1.2,2.5)$ & 0.272 \\
\hline CAD GRS $^{\mathrm{d}}$ & $7.5(7.2,7.9)$ & $7.5(7.2,7.9)$ & 0.471 \\
\hline
\end{tabular}

Values are reported as $n(\%)$, means $\pm \mathrm{SD}$ or median (interquartile range)

$p$ values for continuous measures based on analysis of variance or Kruskal-Wallis tests as appropriate. For categorical measures, $p$ values based on $\chi^{2}$ tests or Fisher's exact test as appropriate

${ }^{a}$ Genetically defined based on estimated admixture proportions (see Methods)

${ }^{\mathrm{b}}$ Available in 2,046 ILI participants and 1,966 DSE participants

${ }^{\mathrm{c}}$ Available in 2,044 ILI participants and 1,968 DSE participants

${ }^{\mathrm{d}}$ Based on reported risk alleles and log-odds ratios reported by the CARDIoGRAMplusC4D consortium (2013). For individual SNP weights, see ESM Table 1
American participants but not African-American participants. The GRS was further associated positively with
SBP, DBP and LDL-cholesterol but inversely with $\mathrm{HbA}_{1 \mathrm{c}}$ level (Table 3). 
Table 2 Distribution of CAD GRS by history of CVD and genetically defined ancestral group

\begin{tabular}{|c|c|c|c|c|c|}
\hline \multirow[t]{2}{*}{ Group } & \multicolumn{2}{|c|}{ No history of CVD } & \multicolumn{2}{|c|}{ History of CVD } & \multirow[t]{2}{*}{$p$ value $^{\mathrm{b}}$} \\
\hline & $n$ & Median (IQR) ${ }^{\mathrm{a}}$ & $n$ & Median (IQR) & \\
\hline All participants & 3,439 & $7.50(7.17,7.85)$ & 577 & $7.65(7.30,7.98)$ & $6.93 \times 10^{-9}$ \\
\hline European ancestry & 2,367 & $7.62(7.30,7.95)$ & 440 & $7.72(7.41,8.03)$ & $1.52 \times 10^{-4}$ \\
\hline African-American & 573 & $7.15(6.89,7.41)$ & 70 & $7.25(7.04,7.41)$ & 0.163 \\
\hline Hispanic/NA & 499 & $7.40(7.06,7.76)$ & 67 & $7.66(7.37,7.92)$ & $2.07 \times 10^{-4}$ \\
\hline
\end{tabular}

${ }^{\text {a }}$ Based on reported risk alleles and log-odds ratios reported by the CARDIoGRAMplusC4D consortium (2013). For individual SNP weights, see ESM Table 1

${ }^{\mathrm{b}} p$ values based on Wilcoxon Rank-Sum test comparing no history of CVD to history of CVD within each population group

NA, Native American; IQR, interquartile range

\section{GRS and CVD outcomes}

Event rates of the primary and secondary composite outcomes in the genetic sub-study of Look AHEAD are presented in ESM Table 2. Prospective associations of the CAD GRS with these primary and secondary composite outcomes of Look AHEAD are presented in Table 4. Across all participants, the GRS significantly predicted the primary composite endpoint of death from cardiovascular causes, nonfatal MI, nonfatal stroke or hospitalisation for angina (HR, 95\% CI per 1 SD increase in GRS: 1.19 [1.10, 1.28]; Fig. 1). Comparing the highest risk GRS quartile to the lowest, a $51 \%$ greater incidence of CVD at 10 years of follow-up (absolute risk

Table 3 Relationship between CAD GRS and risk factors for CVD at baseline in all participants

\begin{tabular}{lcl}
\hline Variable & Estimate $(95 \% \mathrm{CI})^{\mathrm{a}}$ & $p$ value \\
\hline $\mathrm{BMI}\left(\mathrm{kg} / \mathrm{m}^{2}\right)$ & $-0.078(-0.257,0.101)$ & 0.396 \\
$\mathrm{LDL}(\mathrm{mmol} / \mathrm{l})$ & $0.037(0.010,0.065)$ & 0.007 \\
$\mathrm{HDL}(\mathrm{mmol} / \mathrm{l})$ & $-0.005(-0.015,0.005)$ & 0.333 \\
$\mathrm{SBP}(\mathrm{mmHg})$ & $1.462(0.696,2.227)$ & $1.87 \times 10^{-4}$ \\
$\mathrm{DBP}(\mathrm{mmHg})$ & $0.794(0.419,1.168)$ & $3.32 \times 10^{-5}$ \\
Triacylglycerol $(\mathrm{mmol} / \mathrm{l})^{\mathrm{b}}$ & $1.004(0.984,1.024)$ & 0.714 \\
$\mathrm{HbA}_{1 \mathrm{c}}(\%)^{\mathrm{b}}$ & $0.993(0.988,0.998)$ & 0.008 \\
$\mathrm{HbA}_{\mathrm{lc}}(\mathrm{mmol} / \mathrm{mol})^{\mathrm{b}}$ & $0.990(0.983,0.997)$ & 0.008 \\
\hline
\end{tabular}

${ }^{a}$ Adjusted mean change per 1 SD increase in GRS based on linear regression model, with GRS standardised ([GRS-mean]/SD) separately within each ancestral group (European ancestry, African-Americans and Hispanic/Native Americans). All models adjusted for age, sex, clinical site and top three MDS vectors. To address treatment effects of antihypertensive medications on SBP and DBP, lipid-lowering medications on LDL, HDL and triacylglycerol, and insulin use on $\mathrm{HbA}_{1 \mathrm{c}}$, estimates are based on a censored linear regression model, treating the observed values as right-censored for those on treatment for all measures except HDL, which was treated as left-censored

${ }^{\mathrm{b}}$ Modelled on log scale, effects represent multiplicative effects on mean per $1 \mathrm{SD}$ increase in GRS difference $=7.4 \%$ ) was observed. Consistent effects were observed among non-Hispanic white participants (HR 1.17 [95\% CI 1.07, 1.28]) and Hispanic and Native American participants (HR 1.38 [95\% CI 1.08, 1.77]) but not AfricanAmericans. In no case did the magnitude of genetic association differ across the ILI and DSE intervention arms for the primary outcome (interaction $p>0.47$ ).

Similar results were observed for secondary outcome 1, which comprised death from cardiovascular causes, nonfatal MI or nonfatal stroke (full sample: HR 1.16 [95\% CI 1.05, 1.27]; non-Hispanic white participants: 1.13 [1.01, 1.26]; and Hispanic and Native American participants: 1.36 [1.01, 1.82]). In addition, similar findings were observed for secondary outcome 2, which comprised death from any cause, nonfatal MI or nonfatal stroke (full sample: HR 1.16 [95\% CI 1.08, 1.24]; non-Hispanic white participants: 1.11 [1.03, 1.20]; and Hispanic and Native American participants: 1.32 [1.06, 1.64]), and for the broader secondary outcome 3 , which comprised death from any cause, nonfatal MI, nonfatal stroke, hospitalisation for angina, CABG, PCI, hospitalisation for heart failure, carotid endarterectomy or peripheral vascular disease (full sample: HR 1.14 [95\% CI 1.07, 1.21]; non-Hispanic white participants: $1.14[1.05,1.23]$ and Hispanic and Native American participants: 1.34 [1.10, 1.64]). The interaction of the GRS with treatment arm did not approach statistical significance for any of the secondary outcomes in the full sample or in any of the racial and ethnic subgroups $(p>0.25)$.

Given the strong association with CVD at baseline, the prospective association of the GRS with cardiovascular endpoints among individuals without a known history of CVD at baseline was examined (Table 5). The GRS significantly predicted the primary composite endpoint of death from cardiovascular causes, nonfatal MI, nonfatal stroke, or hospitalisation for angina even among individuals with no known history of CVD at baseline (full sample: HR 1.18 [95\% CI 1.07, 1.30]; nonHispanic white participants: $1.15[1.02,1.28]$ and Hispanic and Native American participants: 1.59 [1.18, 2.13]). Similar but more marginal associations were seen for each of the 
Table 4 Association of CAD GRS with primary and secondary composite endpoints

\begin{tabular}{|c|c|c|c|c|c|c|}
\hline \multirow[t]{2}{*}{ Outcome } & \multirow[t]{2}{*}{ Group } & \multicolumn{3}{|c|}{ Stratified by intervention arm } & \multicolumn{2}{|l|}{ Combined cohort } \\
\hline & & $\begin{array}{l}\text { DSE } \\
\text { HR }(95 \% \mathrm{CI})\end{array}$ & $\begin{array}{l}\text { ILI } \\
\text { HR }(95 \% \text { CI) }\end{array}$ & $\begin{array}{l}\text { Interaction } \\
p \text { value }\end{array}$ & HR $(95 \%$ CI $)$ & $p$ value \\
\hline \multirow[t]{4}{*}{ Primary outcome } & All participants ${ }^{\mathrm{a}}$ & $1.16(1.04,1.29)$ & $1.22(1.10,1.36)$ & 0.477 & $1.19(1.10,1.28)$ & $9.50 \times 10^{-6}$ \\
\hline & European ancestry ${ }^{\mathrm{b}}$ & $1.14(1.01,1.28)$ & $1.21(1.06,1.37)$ & 0.510 & $1.17(1.07,1.28)$ & $4.83 \times 10^{-4}$ \\
\hline & African-Americans ${ }^{\mathrm{b}}$ & $1.09(0.80,1.51)$ & $1.15(0.89,1.49)$ & 0.806 & $1.13(0.92,1.38)$ & 0.236 \\
\hline & Hispanic/NA ${ }^{\mathrm{b}}$ & $1.41(0.98,2.03)$ & $1.36(0.97,1.91)$ & 0.888 & $1.38(1.08,1.77)$ & 0.009 \\
\hline \multirow[t]{4}{*}{ Secondary outcome 1} & All participants ${ }^{\mathrm{a}}$ & $1.11(0.97,1.26)$ & $1.21(1.06,1.38)$ & 0.346 & $1.16(1.05,1.27)$ & 0.002 \\
\hline & European ancestry ${ }^{\mathrm{b}}$ & $1.08(0.93,1.25)$ & $1.19(1.01,1.39)$ & 0.378 & $1.13(1.01,1.26)$ & 0.029 \\
\hline & African-Americans ${ }^{\mathrm{b}}$ & $1.00(0.69,1.46)$ & $1.18(0.87,1.59)$ & 0.515 & $1.10(0.87,1.40)$ & 0.411 \\
\hline & Hispanic/NA ${ }^{\mathrm{b}}$ & $1.46(0.95,2.25)$ & $1.28(0.85,1.91)$ & 0.658 & $1.36(1.01,1.82)$ & 0.041 \\
\hline \multirow[t]{4}{*}{ Secondary outcome 2} & All participants ${ }^{\mathrm{a}}$ & $1.12(1.01,1.23)$ & $1.20(1.08,1.32)$ & 0.325 & $1.16(1.08,1.24)$ & $4.51 \times 10^{-5}$ \\
\hline & European ancestry ${ }^{\mathrm{b}}$ & $1.07(0.96,1.19)$ & $1.16(1.05,1.29)$ & 0.258 & $1.11(1.03,1.20)$ & 0.004 \\
\hline & African-Americans ${ }^{\mathrm{b}}$ & $1.07(0.81,1.42)$ & $1.17(0.92,1.48)$ & 0.644 & $1.12(0.94,1.35)$ & 0.212 \\
\hline & Hispanic/NA ${ }^{\mathrm{b}}$ & $1.38(0.99,1.91)$ & $1.27(0.94,1.71)$ & 0.719 & $1.32(1.06,1.64)$ & 0.013 \\
\hline \multirow[t]{4}{*}{ Secondary outcome 3} & All participants ${ }^{\mathrm{a}}$ & $1.10(1.01,1.21)$ & $1.17(1.07,1.28)$ & 0.356 & $1.14(1.07,1.21)$ & $9.84 \times 10^{-5}$ \\
\hline & European ancestry ${ }^{\mathrm{b}}$ & $1.10(0.98,1.22)$ & $1.18(1.06,1.33)$ & 0.342 & $1.14(1.05,1.23)$ & 0.002 \\
\hline & African-Americans ${ }^{\mathrm{b}}$ & $1.12(0.87,1.44)$ & $1.07(0.85,1.34)$ & 0.796 & $1.09(0.92,1.29)$ & 0.314 \\
\hline & Hispanic/NA ${ }^{\mathrm{b}}$ & $1.38(1.02,1.86)$ & $1.31(1.00,1.73)$ & 0.807 & $1.34(1.10,1.64)$ & 0.004 \\
\hline
\end{tabular}

Primary composite outcome includes death from cardiovascular causes, nonfatal myocardial infarction, nonfatal stroke, or hospitalisation for angina. Secondary outcome 1 includes death from cardiovascular causes, nonfatal myocardial infarction or nonfatal stroke. Secondary outcome 2 includes death from any cause, nonfatal myocardial infarction or nonfatal stroke. Secondary outcome 3 includes death from any cause, nonfatal myocardial infarction, nonfatal stroke, hospitalisation for angina, CABG, PCI, hospitalisation for heart failure, carotid endarterectomy or peripheral vascular disease. Reported HRs reflect change per 1 SD increase in GRS, with SDs computed separately within each ancestral group (European ancestry, African-Americans and Hispanic/NA)

${ }^{a}$ Estimates adjusted for age, sex, treatment assignment (DSE or ILI), top three MDS vectors and clinic (gamma frailty)

${ }^{\mathrm{b}}$ Estimates adjusted for age, sex, treatment assignment (DSE or ILI) and clinic (gamma frailty)

NA, Native American

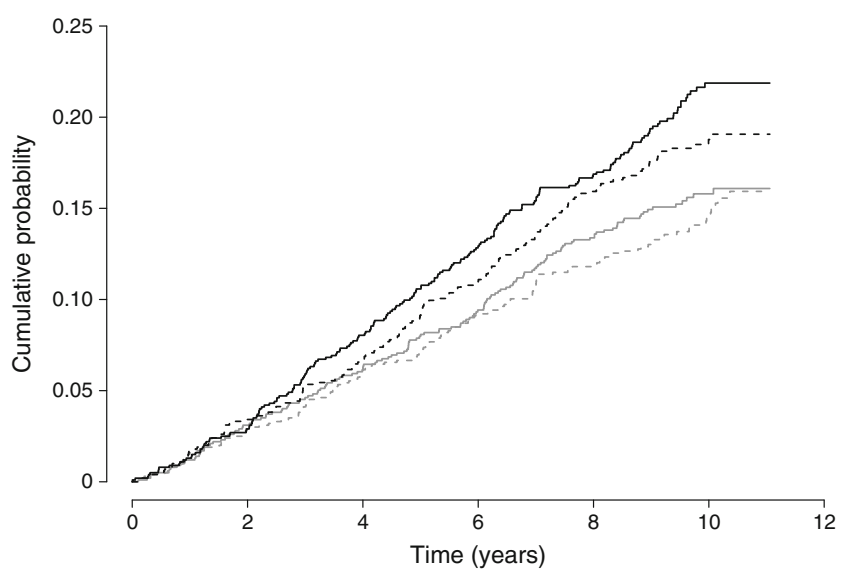

Fig. 1 Kaplan-Meier estimates for primary composite endpoint by GRS quartiles. GRS quartiles were defined after standardising GRS ([GRS - mean]/SD) separately within each ancestral group (European ancestry, African-Americans and Hispanic/Native Americans). First (lowest genetic risk) quartile, grey dashed line; second quartile, grey solid line; third quartile, black dashed line; fourth quartile, black solid line. Primary composite outcome includes death from cardiovascular causes, nonfatal myocardial infarction, nonfatal stroke or hospitalisation for angina secondary outcomes in this subgroup. No significant interactions with the treatment arm were observed $(p>0.29)$.

The observed association of the GRS with the primary endpoint of the trial persisted after further statistical adjustment for cardiometabolic risk factors as well as use of aspirin and antihypertensive and lipid-lowering medications in the full sample (HR 1.17 [95\% CI 1.09, 1.27]) and among participants without a known history of CVD at baseline (HR 1.17 [95\% CI 1.06, 1.29]). Similar but weaker associations with the secondary outcomes were observed. Interactions of GRS with the treatment arm remained nonsignificant after further adjustment for cardiovascular risk factors and medication use $(p>0.37)$.

\section{Discussion}

The evaluation of GRS in the context of prospective treatment trials is critical to the clinical application of new discoveries in cardiovascular genetics. This paper tested whether a GRS constructed using the most comprehensive list of SNPs to date [4] contributes to incident CVD as defined by the adjudicated 
Table 5 Association of CAD GRS with primary and secondary composite endpoints in participants without a history of CVD at baseline

\begin{tabular}{|c|c|c|c|c|c|c|}
\hline \multirow[t]{2}{*}{ Outcome } & \multirow[t]{2}{*}{ Group } & \multicolumn{3}{|c|}{ Stratified by intervention arm } & \multicolumn{2}{|l|}{ Combined cohort } \\
\hline & & $\begin{array}{l}\text { DSE } \\
\text { HR }(95 \% \mathrm{CI})\end{array}$ & $\begin{array}{l}\text { ILI } \\
\text { HR }(95 \% \text { CI) }\end{array}$ & $\begin{array}{l}\text { Interaction } \\
p \text { value }\end{array}$ & HR $(95 \%$ CI $)$ & $p$ value \\
\hline \multirow[t]{4}{*}{ Primary outcome } & All participants ${ }^{\mathrm{a}}$ & $1.15(1.01,1.31)$ & $1.22(1.06,1.40)$ & 0.575 & $1.18(1.07,1.30)$ & $6.89 \times 10^{-4}$ \\
\hline & European ancestry ${ }^{\mathrm{b}}$ & $1.13(0.97,1.31)$ & $1.17(0.98,1.38)$ & 0.778 & $1.15(1.02,1.28)$ & 0.018 \\
\hline & African-Americans ${ }^{\mathrm{b}}$ & $1.02(0.70,1.50)$ & $1.10(0.83,1.47)$ & 0.758 & $1.07(0.85,1.35)$ & 0.555 \\
\hline & Hispanic/NA ${ }^{\mathrm{b}}$ & $1.44(0.96,2.17)$ & $1.77(1.14,2.73)$ & 0.505 & $1.59(1.18,2.13)$ & 0.002 \\
\hline \multirow[t]{4}{*}{ Secondary outcome 1} & All participants ${ }^{\mathrm{a}}$ & $1.14(0.97,1.34)$ & $1.20(1.01,1.43)$ & 0.674 & $1.17(1.04,1.31)$ & 0.011 \\
\hline & European ancestry ${ }^{\mathrm{b}}$ & $1.13(0.94,1.36)$ & $1.16(0.93,1.44)$ & 0.869 & $1.14(0.99,1.31)$ & 0.069 \\
\hline & African-Americans ${ }^{\mathrm{b}}$ & $0.88(0.57,1.37)$ & $1.19(0.84,1.67)$ & 0.296 & $1.06(0.81,1.39)$ & 0.672 \\
\hline & Hispanic/NA ${ }^{\mathrm{b}}$ & $1.56(0.96,2.53)$ & $1.38(0.81,2.34)$ & 0.732 & $1.48(1.03,2.11)$ & 0.032 \\
\hline \multirow[t]{4}{*}{ Secondary outcome 2} & All participants ${ }^{\mathrm{a}}$ & $1.08(0.97,1.22)$ & $1.16(1.03,1.32)$ & 0.404 & $1.12(1.03,1.22)$ & 0.008 \\
\hline & European ancestry ${ }^{\mathrm{b}}$ & $1.04(0.91,1.18)$ & $1.13(0.99,1.29)$ & 0.381 & $1.08(0.98,1.18)$ & 0.104 \\
\hline & African-Americans ${ }^{\mathrm{b}}$ & $0.93(0.66,1.29)$ & $1.11(0.85,1.45)$ & 0.396 & $1.04(0.84,1.28)$ & 0.739 \\
\hline & Hispanic/NA ${ }^{\mathrm{b}}$ & $1.40(0.98,2.00)$ & $1.50(1.02,2.20)$ & 0.802 & $1.44(1.11,1.87)$ & 0.005 \\
\hline \multirow[t]{4}{*}{ Secondary outcome 3} & All participants ${ }^{\mathrm{a}}$ & $1.07(0.96,1.20)$ & $1.13(1.01,1.27)$ & 0.501 & $1.10(1.02,1.19)$ & 0.015 \\
\hline & European ancestry ${ }^{\mathrm{b}}$ & $1.07(0.94,1.22)$ & $1.13(0.97,1.31)$ & 0.605 & $1.10(1.00,1.21)$ & 0.063 \\
\hline & African-Americans ${ }^{\mathrm{b}}$ & $1.04(0.78,1.38)$ & $1.00(0.77,1.29)$ & 0.844 & $1.01(0.84,1.23)$ & 0.882 \\
\hline & Hispanic/NA ${ }^{\mathrm{b}}$ & $1.39(1.00,1.94)$ & $1.46(1.03,2.08)$ & 0.849 & $1.43(1.12,1.81)$ & 0.004 \\
\hline
\end{tabular}

Primary composite outcome includes death from cardiovascular causes, nonfatal myocardial infarction, nonfatal stroke, or hospitalisation for angina. Secondary outcome 1 includes death from cardiovascular causes, nonfatal myocardial infarction or nonfatal stroke. Secondary outcome 2 includes death from any cause, nonfatal myocardial infarction or nonfatal stroke. Secondary outcome 3 includes death from any cause, nonfatal myocardial infarction, nonfatal stroke, hospitalisation for angina, CABG, PCI, hospitalisation for heart failure, carotid endarterectomy or peripheral vascular disease. Reported HRs reflect change per 1 SD increase in GRS, with SDs computed separately within each ancestral group (European ancestry, African-Americans and Hispanic/NA).

${ }^{a}$ Estimates adjusted for age, sex, treatment assignment (DSE or ILI), top three MDS vectors and clinic (gamma frailty)

${ }^{\mathrm{b}}$ Estimates adjusted for age, sex, treatment assignment (DSE or ILI) and clinic (gamma frailty)

NA, Native American

primary and secondary outcomes of Look AHEAD among overweight or obese participants with type 2 diabetes. We further determined whether such an association may be diminished by a behavioural weight-loss treatment.

We consider there to be three key findings in this paper. First, the GRS was prospectively associated with adjudicated CVD morbidity and mortality in the setting of type 2 diabetes and obesity in Look AHEAD. Findings from CARDIoGRAMplusC4D consortia indicated that these SNPs accounted for $10.6 \%$ of the additive genetic variance in CAD. By using the longitudinal design, we moreover found a $51 \%$ greater incidence of CVD at a median of 9.6 years of follow-up (absolute risk difference $=7.4 \%$ ), comparing the highest risk GRS quartile with the lowest risk GRS quartile in Look AHEAD. The present results extend prior research by incorporating the most comprehensive list of SNPs and demonstrating prospective association in the context of mixed ancestry as well as diabetes. The consistency of the findings across multiple primary and secondary endpoints that included myocardial infarction, stroke and coronary artery revascularisation also suggests that this assessment of genetic risk, derived in relation to CAD, can be applied more broadly to composite endpoints that include other systemic manifestations of atherosclerosis.

Not surprisingly, the GRS was most strongly associated with incident CVD in non-Hispanic white participants, as samples contributing to CARDIoGRAMplusC4D consortia were largely selected for European ancestry. Intriguingly, we observed a consistent association in Hispanic and Native American Look AHEAD participants, despite the small sample size, but no association among African-Americans. This finding extends emerging research on genetic associations with CVD morbidity and mortality by including under-represented minorities $[10,11]$, but more definitive research is needed.

The second principal finding was the association of the GRS with traditional risk factors of CVD, including LDL-cholesterol, SBP and DBP. Association of the GRS with lipoprotein levels in Look AHEAD is consistent with the CARDIoGRAMplusC4D consortia results that demonstrated that nearly all variants included in the GRS were 
associated with one or more lipid traits. Moreover, while only a select number of individual SNPs were associated with blood pressure, here, we identify an association of the GRS with both SBP and DBP. Interestingly, while the CARD IoGRAMplusC4D consortia did not find an association of the individual variants with type 2 diabetes, we identified an inverse association of the GRS with $\mathrm{HbA}_{1 \mathrm{c}}$ level.

Finally, the behavioural intervention did not alter the association of the GRS with the primary and secondary endpoints of Look AHEAD. This is despite the demonstrated efficacy of the intervention in reducing weight and producing improvements in fitness, waist circumference, HDL, triacylglycerol, $\mathrm{SPB}, \mathrm{DBP}$ and $\mathrm{HbA}_{1 \mathrm{c}}$ level at 1 year [32], with intervention effects on weight, waist circumference and $\mathrm{HbA}_{1 \mathrm{c}}$ level continuing across a median of 9.6 years of follow-up [15]. Thus, taking genetic vulnerability into account did not help to explain why effective lifestyle intervention did not reduce cardiovascular morbidity and mortality among overweight individuals with type 2 diabetes. In some respects this finding is understandable because the GRS was constructed using data from 63,746 CAD cases and 130,681 controls who probably had a wide range of behavioural and fitness practices [4]. Furthermore, although obesity genetic risk loci have been identified, none of these were identified in the CARD IoGRAMplusC4D consortia as being associated with CAD.

Look AHEAD is the largest randomised controlled trial of behavioural weight loss, with over 4,000 individuals with genetic consent randomly assigned to either ILI focusing on weight loss and physical activity promotion or a minimal contact control group. Prior genetic research in Look AHEAD indicated that obesity-associated SNPs, such as those within $F T O$ and $B D N F$, were associated with an obesogenic pattern of dietary intake [33]; the same SNPs within FTO predicted weight regain [34]. With regard to lipids, several SNPs within CETP, LIPC and FADS2 modified the association of behavioural weight loss treatment with changes in HDL [35]. In this first study of CVD morbidity and mortality, a GRS comprising SNPs previously associated with CAD (but with little overlap with previously analysed SNPs associated with obesity or HDL) was associated with a $51 \%$ greater incidence of CVD at 10 years of follow-up, comparing the highest risk GRS quartile with the lowest risk GRS quartile. However, the effect was largely invariant when compared across ILI and DSE arms. It remains plausible that behavioural weight loss could reduce the genetic risk of CVD among individuals without an advanced disease state, such as diabetes, or among those not using medications to manage obesity-related comorbidities including dysglycaemia and elevated cholesterol and blood pressure.

This study has both strengths and limitations. Look AHEAD had excellent retention rates over nearly 10 years and cardiovascular outcomes were adjudicated by reviewers masked to treatment assignment. The study had good power to detect reasonably small GRS main effects and larger GRS $\times$ treatment arm interactions. Although power was more limited to detect interactions of smaller effect size, we note that the GRS increased risk in both ILI and DSE, suggesting that the treatment arm did not appear to substantially mitigate the association of the GRS with CVD morbidity and mortality and that the small qualitative interaction, if detectable in a larger sample size, would be unlikely to be clinically significant. While SNPs studied here were selected on the basis of their prior association with CAD in the CARDIoGRAMplusC4D consortia, we cannot exclude the possibility that there are other important gene variants that influence CVD and response to behavioural intervention. Although our findings from Look AHEAD may apply to a growing population of individuals with type 2 diabetes, our findings may not be generalisable to a non-diabetic population. Finally, as the vast majority of the population used diabetes medications, we were unable to consider this variable in analyses.

Overall, our findings build upon prior research by demonstrating prospective association of the most comprehensive GRS to date with incident cardiovascular morbidity and mortality over a median of 9.6 years follow-up among overweight or obese individuals with type 2 diabetes. This prospective association was not altered by lifestyle intervention promoting weight loss and physical activity.

Acknowledgements Authors: Jeanne M. McCaffery (Department of Psychiatry and Human Behavior, The Miriam Hospital/Alpert School of Medicine at Brown University, Providence, RI, USA), Andrea Anderson (Department of Biostatistical Sciences, Wake Forest School of Medicine, Winston-Salem, NC, USA), John P. Bantle (Department of Medicine, University of Minnesota, Minneapolis, MN, USA), Robert I. Berkowitz (Department of Psychiatry, Perelman School of Medicine, University of Pennsylvania, Philadelphia, PA, USA), George A. Bray (Department of Clinical Obesity, Pennington Biomedical Research Center, Louisiana State University, Baton Rouge, LA, USA), Lawrence Cheskin (Health, Behavior and Society; The Global Obesity Prevention Center at Johns Hopkins, Johns Hopkins Bloomberg School of Public Health, Baltimore, MD, USA), Jeanne M. Clark (Department of Medicine [General Internal Medicine] and Epidemiology, The Johns Hopkins University, Baltimore, MD, USA), Jeffrey M. Curtis (Department of Family Medicine, St. Joseph's Hospital and Medical Center, Phoenix, AZ, USA; Southwest American Indian Center, National Institute for Diabetes and Digestive and Kidney Disease, National Institutes for Health, Phoenix, AZ, USA), Linda M. Delahanty (Department of Medicine, Massachusetts General Hospital Diabetes Center and Harvard Medical School, Boston, MA, USA), Mary Evans (Department of Digestive Diseases and Nutrition, National Institute of Diabetes and Digestive and Kidney Diseases, Bethesda, MD, USA), John P. Foreyt (Department of Medicine, Baylor College of Medicine, Houston, TX, USA), Stephen Glasser (Department of Medicine/Division of Preventive Medicine, University of Alabama at Birmingham, Birmingham, AL, USA), Edward W. Gregg (Division of Diabetes Translation, Centers for Disease Control and Prevention, Atlanta, GA, USA), Robert L. Hanson (Diabetes Epidemiology and Clinical Research Section, National Institute of Diabetes and Digestive and Kidney Diseases, Phoenix, AZ, USA), Helen P. Hazuda (Department of Medicine, Division of Nephrology, University of Texas Health Science Center at San Antonio, TX, USA), James O. Hill (Anschutz Health and Wellness Center, University of Colorado, Aurora, 
CO, USA), Edward S. Horton (Joslin Diabetes Center, Boston, MA, USA; Department of Medicine, Harvard Medical School, Boston, MA, USA), Gordon S. Huggins (MCRI Center for Translational Genomics, Tufts Medical Center, Boston, MA USA), John M. Jakicic (University of Pittsburgh, Department of Health and Physical Activity, Pittsburgh, PA, USA), Robert W. Jeffery (Division of Epidemiology and Community Health, School of Public Health, University of Minnesota, Minneapolis, MN, USA), Karen C. Johnson (Department of Preventive Medicine. University of Tennessee Health Science Center, Memphis, TN, USA), Steven E. Kahn (Division of Metabolism, Endocrinology and Nutrition, Department of Medicine, VA Puget Sound Health Care System and University of Washington, Seattle, WA, USA), David E. Kelley (University of Pittsburgh, Department of Health and Physical Activity, Pittsburgh, PA, USA), Abbas E. Kitabchi (Department of Medicine, University of Tennessee Health Science Center, Memphis, TN, USA), William C. Knowler (National Institute of Diabetes and Digestive and Kidney Diseases, Phoenix, AZ, USA), Cora E. Lewis (Division of Preventive Medicine, Dept of Medicine, University of Alabama at Birmingham, Birmingham, AL, USA), Maria G. Montez (Department of Medicine, Division of Nephrology, University of Texas Health Science Center at San Antonio, TX, USA), Anne Kure (VA Puget Sound Health Care System and University of Washington, Seattle, WA, USA), David M. Nathan (Diabetes Unit, Department of Medicine, Massachusetts General Hospital Diabetes Center and Harvard Medical School, Boston, MA, USA), Ebenezer Nyenwe (Division of Endocrinology, Diabetes \& Metabolism, University of Tennessee Health Science Center, Memphis, TN, USA), Nicholas M. Pajewski (Department of Biostatistical Sciences, Wake Forest School of Medicine, Winston-Salem, NC, USA), George D. Papandonatos (Department of Biostatistics, School of Public Health, Brown University, Providence, RI, USA), Jennifer Patricio (New York Obesity Research Center, St. Luke's-Roosevelt Hospital Center, New York, NY, USA), Inga Peter (Dept. of Genetics \& Genomic Sciences, Mount Sinai School of Medicine, New York, NY USA), Anne Peters (Roybal Comprehensive Health Center, Los Angeles, CA, USA), Xavier Pi-Sunyer (New York Obesity Research Center, St. Luke's-Roosevelt Hospital Center, New York, NY, USA), Henry Pownall (Department of Cardiology, Methodist Hospital Research Institute, Houston, TX, USA), Thomas A. Wadden (Department of Psychiatry, Perelman School of Medicine at the University of Pennsylvania, Philadelphia, PA, USA.), Lynne E. Wagenknecht (Department of Public Health Services, Wake Forest School of Medicine, Winston-Salem, NC, USA), Rena R. Wing (Department of Psychiatry and Human Behavior, The Miriam Hospital/Alpert School of Medicine at Brown University, Providence, RI, USA) and Holly Wyatt (Anschutz Health and Wellness Center, University of Colorado, Aurora, CO, USA). Please see ESM for full listing of Look AHEAD investigators.

Funding Funded by the National Institutes of Health through cooperative agreements with the National Institute of Diabetes and Digestive and Kidney Diseases: DK57136, DK57149, DK56990, DK57177, DK57171, DK57151, DK57182, DK57131, DK57002, DK57078, DK57154, DK57178, DK57219, DK57008, DK57135 and DK56992. Additional funding was provided by the National Heart, Lung, and Blood Institute; National Institute of Nursing Research; National Center on Minority Health and Health Disparities; NIH Office of Research on Women's Health; and the Centers for Disease Control and Prevention. This research was supported in part by the Intramural Research Program of the National Institute of Diabetes and Digestive and Kidney Diseases. The Indian Health Service (I.H.S.) provided personnel, medical oversight and use of facilities. The opinions expressed in this paper are those of the authors and do not necessarily reflect the views of the I.H.S. or other funding sources.

Additional support was received from The Johns Hopkins Medical Institutions Bayview General Clinical Research Center (M01RR02719); the Massachusetts General Hospital Mallinckrodt General Clinical Research Center and the Massachusetts Institute of Technology General
Clinical Research Center (M01RR01066); the University of Colorado Health Sciences Center General Clinical Research Center (M01RR00051) and Clinical Nutrition Research Unit (P30 DK48520); the University of Tennessee at Memphis General Clinical Research Center (M01RR0021140); the University of Pittsburgh General Clinical Research Center (GCRC) (M01RR000056); the Clinical Translational Research Center (CTRC) funded by the Clinical and Translational Science Award (UL1 RR 024153) and NIH grant (DK 046204); the VA Puget Sound Health Care System Medical Research Service, Department of Veterans Affairs; and the Frederic C. Bartter General Clinical Research Center (M01RR01346).

The following organisations have committed to make major contributions to Look AHEAD: FedEx Corporation; Health Management Resources; LifeScan, Inc., a Johnson \& Johnson Company; OPTIFAST ${ }^{\circledR}$ of Nestle HealthCare Nutrition, Inc.; Hoffmann-La Roche Inc.; Abbott Nutrition; and Slim-Fast Brand of Unilever North America.

Some of the information contained herein was derived from data provided by the Bureau of Vital Statistics, New York City Department of Health and Mental Hygiene.

Duality of interest The authors declare that there is no duality of interest associated with this manuscript.

Contribution statement JMM, NMP, GSH, WCK, RRW, LEW, KCJ, CEL, GDP, IP, LMD and SEK wrote the first draft of the manuscript. GAB, LC, JMClark, ME, CEL, JOH, DMN, JPF, ESH, KCJ, AEK, JMJ, RWJ, SEK, XP-S, TAW, AP, HPH and EWG contributed to the funding of the study and to its conception and design. GAB, LC, JMClark, ME, CEL, JOH, DMN, JPF, ESH, KCJ, AEK, JMJ, RWJ, SEK, XP-S, TAW, AP, HPH, EWG, AA, JPB, RIB, JMCurtis, SG, RLH, DEK, MGM, AK, EN, JP, HP, LMD and HW contributed to the acquisition of data. NMP contributed to the data analysis. JMM, NMP, GSH, WCK, RRW, LEW, KCJ, CEL, GDP, IP, LMD and SEK contributed to data interpretation. All authors reviewed and edited the manuscript and gave final approval of the version to be published. JMM is responsible for the integrity of the work as a whole.

\section{References}

1. Go AS, Mozaffarian D, Roger VL et al (2014) Heart disease and stroke statistics-2014 update: a report from the American Heart Association. Circulation 129:e28-e292

2. Lopez AD, Mathers CD, Ezzati M, Jamison DT, Murray CJ (2006) Global and regional burden of disease and risk factors, 2001: systematic analysis of population health data. Lancet 367:1747-1757

3. Ford ES, Ajani UA, Croft JB et al (2007) Explaining the decrease in U.S. deaths from coronary disease, 1980-2000. N Engl J Med 356: 2388-2398

4. Deloukas P, Kanoni S, Willenborg C et al (2013) Large-scale association analysis identifies new risk loci for coronary artery disease. Nat Genet 45:25-33

5. Weijmans M, de Bakker PI, van der Graaf Y et al (2015) Incremental value of a genetic risk score for the prediction of new vascular events in patients with clinically manifest vascular disease. Atherosclerosis 239:451-458

6. Brautbar A, Pompeii LA, Dehghan A et al (2012) A genetic risk score based on direct associations with coronary heart disease improves coronary heart disease risk prediction in the Atherosclerosis Risk in Communities (ARIC), but not in the Rotterdam and Framingham Offspring, Studies. Atherosclerosis 223:421-426

7. Isaacs A, Willems SM, Bos D et al (2013) Risk scores of common genetic variants for lipid levels influence atherosclerosis and 
incident coronary heart disease. Arterioscler Thromb Vasc Biol 33: 2233-2239

8. Thanassoulis G, Peloso GM, Pencina MJ et al (2012) A genetic risk score is associated with incident cardiovascular disease and coronary artery calcium - The Framingham Heart Study. Circ Cardiovasc Genet 5:113-121

9. Patel RS, Sun YV, Hartiala J et al (2012) Association of a genetic risk score with prevalent and incident myocardial infarction in subjects undergoing coronary angiography. Circ Cardiovasc Genet 5:441-449

10. Qi L, Ma J, Qi Q, Hartiala J, Allayee H, Campos H (2011) Genetic risk score and risk of myocardial infarction in Hispanics. Circulation 123:374-380

11. Franceschini N, Carty C, Buzkova P et al (2011) Association of genetic variants and incident coronary heart disease in multiethnic cohorts: the PAGE study. Circ Cardiovasc Genet 4:661-672

12. Thanassoulis G, Vasan RS (2010) Genetic cardiovascular risk prediction: will we get there? Circulation 122:2323-2334

13. Vaarhorst AA, Lu Y, Heijmans BT et al (2012) Literature-based genetic risk scores for coronary heart disease: the Cardiovascular Registry Maastricht (CAREMA) prospective cohort study. Circ Cardiovasc Genet 5:202-209

14. Pi-Sunyer X, Blackburn G, Brancati FL et al (2007) Reduction in weight and cardiovascular disease risk factors in individuals with type 2 diabetes: one-year results of the look AHEAD trial. Diabetes Care 30:1374-1383

15. Wing RR, Bolin P, Brancati FL et al (2013) Cardiovascular effects of intensive lifestyle intervention in type 2 diabetes. N Engl J Med 369:145-154

16. Ryan DH, Espeland MA, Foster GD et al (2003) Look AHEAD (Action for Health in Diabetes): design and methods for a clinical trial of weight loss for the prevention of cardiovascular disease in type 2 diabetes. Control Clin Trials 24:610-628

17. Bray G, Gregg E, Haffner S et al (2006) Baseline characteristics of the randomised cohort from the Look AHEAD (Action for Health in Diabetes) study. Diab Vasc Dis Res 3:202-215

18. Espeland MA, Dotson K, Jaramillo SA et al (2006) Consent for genetics studies among clinical trial participants: findings from Action for Health in Diabetes (Look AHEAD). Clin Trials 3:443456

19. McCaffery JM, Papandonatos GD, Huggins GS et al (2013) Human cardiovascular disease IBC chip-wide association with weight loss and weight regain in the look AHEAD trial. Hum Hered 75:160-174

20. Manichaikul A, Palmas W, Rodriguez CJ et al (2012) Population structure of Hispanics in the United States: the multi-ethnic study of atherosclerosis. PLoS Genet 8, e1002640
21. Purcell S, Neale B, Todd-Brown K et al (2007) PLINK: a tool set for whole-genome association and population-based linkage analyses. Am J Hum Genet 81:559-575

22. Manichaikul A, Mychaleckyj JC, Rich SS, Daly K, Sale M, Chen WM (2010) Robust relationship inference in genome-wide association studies. Bioinformatics 26:2867-2873

23. Alexander DH, Novembre J, Lange K (2009) Fast model-based estimation of ancestry in unrelated individuals. Genome Res 19: 1655-1664

24. Thornton T, Tang H, Hoffmann TJ, Ochs-Balcom HM, Caan BJ, Risch N (2012) Estimating kinship in admixed populations. Am J Hum Genet 91:122-138

25. Staples J, Nickerson DA, Below JE (2013) Utilizing graph theory to select the largest set of unrelated individuals for genetic analysis. Genet Epidemiol 37:136-141

26. Lee SH, Wray NR, Goddard ME, Visscher PM (2011) Estimating missing heritability for disease from genome-wide association studies. Am J Hum Genet 88:294-305

27. Browning BL, Browning SR (2009) A unified approach to genotype imputation and haplotype-phase inference for large data sets of trios and unrelated individuals. Am J Hum Genet 84:210-223

28. Tobin MD, Sheehan NA, Scurrah KJ, Burton PR (2005) Adjusting for treatment effects in studies of quantitative traits: antihypertensive therapy and systolic blood pressure. Stat Med 24:2911-2935

29. Glidden DV, Vittinghoff E (2004) Modelling clustered survival data from multicentre clinical trials. Stat Med 23:369-388

30. Core Team R (2014) R: a language and environment for statistical computing. R Foundation for Statistical Computing, Vienna

31. Bender R, Augustin T, Blettner M (2005) Generating survival times to simulate Cox proportional hazards models. Stat Med 24:1713-1723

32. Wing RR, Lang W, Wadden TA et al (2011) Benefits of modest weight loss in improving cardiovascular risk factors in overweight and obese individuals with type 2 diabetes. Diabetes Care 34:14811486

33. McCaffery JM, Papandonatos GD, Peter I et al (2012) Obesity susceptibility loci and dietary intake in the Look AHEAD trial. Am J Clin Nutr 95:1477-1486

34. McCaffery JM, Papandonatos GD, Huggins GS et al (2013) FTO predicts weight regain in the Look AHEAD clinical trial. Int J Obes (Lond) 37:1545-1552

35. Huggins GS, Papandonatos GD, Erar B et al (2013) Do genetic modifiers of high-density lipoprotein cholesterol and triglyceride levels also modify their response to a lifestyle intervention in the setting of obesity and type-2 diabetes mellitus?: The Action for Health in Diabetes (Look AHEAD) study. Circ Cardiovasc Genet 6:391-399 